\title{
ADESÃO AO CHECKLIST DE CIRURGIA SEGURA: ANÁLISE DAS CIRURGIAS PEDIÁTRICAS
}

\author{
Safe surgery compliance checklist: analysis of pediatric surgeries
}

Adhesión al checklist de cirugía segura: análisis de las cirugías pediátricas

Letícia Costa Rinaldi* (D), Jamille Duran Matilde ${ }^{2}$ (D), Rafaela Aparecida Prata ${ }^{3}$ (D), Andrezza Belluomini Castro ${ }^{4}$, Marla Andréia Garcia de Avila ${ }^{5}$

RESUMO: Objetivo: Este estudo analisou a adesão ao preenchimento do checklist de cirurgia segura em procedimentos realizados em crianças e adolescentes de até 17 anos, bem como os fatores que influenciam a sua utilização. Método: Estudo analítico, transversal, realizado em um hospital público. Foram analisados os prontuários de cirurgias executadas em crianças e adolescentes de até 17 anos, no ano de 2017. Foi aplicada análise estatística descritiva, teste Exato de Fisher e regressão logística. Resultados: A amostra foi composta por 262 prontuários de crianças e adolescentes, 65,68\% do sexo masculino, prevalecendo os procedimentos de adenoidectomia e amigdalectomia. Observou-se em 12,9\% dos checklists o preenchimento completo, em $86,4 \%$, parcial e em $0,7 \%$ a lista não foi preenchida. Não houve associação significativa entre a adesão ao instrumento e os fatores analisados. Conclusão: A adesão completa ao checklist foi de $12,9 \%$ com diferença no preenchimento entre as etapas, e não houve um único fator responsável pela inadequação. O preenchimento parcial na maioria dos casos sinaliza a necessidade de desconstruir as barreiras para conduzir o checklist, com ações educativas envolvendo as equipes e o real entendimento da aplicação do instrumento, que pode favorecer a segurança cirúrgica e a qualidade da assistência.

Palavras-chave: Lista de checagem. Segurança do paciente. Enfermagem perioperatória.

ABSTRACT: Objective: This study analyzed the safe surgical checklist compliance in surgeries performed in children and adolescents up to 17 years old, as well as the factors that influence its use. Method: Cross-sectional, analytical study performed in a public hospital. The medical charts of surgeries performed on children and adolescents up to the age of 17 were analyzed in the year 2017. Descriptive statistical analysis, Fisher's exact test and logistic regression were applied. Results: The sample consisted of 262 medical records of children and adolescents, $65.68 \%$ belonged to males, adenoidectomy and tonsillectomy procedures were prevalent. It was observed that $12.9 \%$ checklists were fully completed, $86.4 \%$ partially completed and $0.7 \%$ were not completed. There was no significant association between compliance to the instrument and the factors analyzed. Conclusion: Complete adherence to the checklist was $12.9 \%$, with differences in completion between stages, and there was no single factor responsible for the inadequacy. The partial completion in most cases indicates the need to deconstruct the barriers related to performing the checklist, by employing educational actions involving the teams and understanding the application of the instrument, which may benefit surgical safety and quality of care.

Keywords: Checklist. Patient safety. Perioperative nursing.

RESUMEN: Objetivo: Este estudio analizó la adhesión al llenado del checklist de cirugía segura en cirugías realizadas en niños y adolescentes de hasta 17 años, así como los factores que influyen su utilización. Método: Estudio analítico, transversal, realizado en un hospital público. Fueron analizados los prontuarios de cirugías ejecutadas en niños y adolescentes de hasta 17 años, el año de 2017. Fue aplicado análisis estadístico descriptivo, test Exacto de Fisher y regresión logística. Resultados: La muestra fue compuesta por 262 históricos médicos de niños y adolescentes, un 65,68\% del sexo masculino,

\footnotetext{
'Enfermeira pela Universidade Estadual Paulista “Júlio de Mesquita Filho" (UNESP) - Botucatu (SP), Brasil.

"Enfermeira. Mestranda em Enfermagem pelo Programa de Pós-Graduação da UNESP (UNESP) - Botucatu (SP), Brasil.

${ }^{3}$ Enfermeira. Doutoranda em Enfermagem pelo Programa de Pós-Graduação da UNESP (UNESP) - Botucatu (SP), Brasil.

${ }^{4}$ Enfermeira. Mestre em Enfermagem pela UNESP - Botucatu (SP), Brasil.

${ }^{5}$ Enfermeira. Mestre em Biotecnologia Médica. Doutora em Saúde Coletiva. Orientadora do Programa de Pós-Graduação em Enfermagem da UNESP Botucatu (SP), Brasil.

*Autor correspondente: leticia.costarinaldi@gmail.com

Fonte de Financiamento: o trabalho foi financiado pela bolsa PIBIC Reitoria da Faculdade de Medicina de Botucatu da Universidade Estadual Paulista - Júlio de Mesquita Filho - (UNESP) - Botucatu (SP), Brasil. Recebido: 13/03/2019 - Aprovado: 13/06/2019

DOI: $10.5327 / Z 1414-4425201900040003$
} 
prevaleciendo los procedimientos de adenoidectomía e amigdalectomía. Se observó en un 12,9\% de los checklists el 1lenado completo, en un 86,4\%, parcial y en un $0,7 \%$ la lista no fue llenada. No hubo asociación significativa entre la adhesión al instrumento y los factores analizados. Conclusión: La adhesión completa al checklist fue del 12,9\% con diferencia en el llenado entre las etapas, y no hubo un único factor responsable por la inadecuación. El llenado parcial en la mayoría de los casos señala la necesidad de deconstruir las barreras para conducir el checklist, con acciones educativas involucrando los equipos y el real entendimiento de la aplicación del instrumento, que puede favorecer la seguridad quirúrgica y la calidad de la asistencia.

Palabras clave: Lista de verificación. Seguridad del paciente. Enfermería perioperatoria.

\section{INTRODUÇÃo}

Com o progresso tecnológico e o aperfeiçoamento da atenção à saúde, a assistência cirúrgica tornou-se uma intervenção com consideráveis possibilidades, amplitude de acesso e alta complexidade, mas passível de erro ${ }^{1,2}$. Os procedimentos cirúrgicos expandiram-se, e anualmente são realizadas cerca de 234 milhões de cirurgias, sendo, em média, uma para cada 25 pessoas, o que gera grande impacto na saúde pública $^{3}$. Entre essas intervenções cirúrgicas, estima-se que todos os anos sete milhões de pacientes sofrem complicações relevantes e ao menos um milhão morrem durante ou após o procedimento ${ }^{3}$.

De acordo com a Organização Mundial da Saúde (OMS), todo ano dezenas de milhões de pacientes sofrem lesões incapacitantes ou morte por efeito da assistência inadequada à saúde, e metade das complicações pós-operatórias é considerada evitável mundialmente ${ }^{3}$. Apesar dos avanços na atenção cirúrgica, os eventos adversos no perioperatório chegam a $3 \%$, sendo mais da metade tida como evitável ${ }^{3}$.

A infraestrutura inadequada, a baixa qualidade dos insumos, a falta de capacitações e a escassez de recursos humanos e de materiais são as circunstâncias primárias que levam aos eventos cirúrgicos adversos ${ }^{1,4}$. Outros fatores, como a não identificação do paciente, a falta de reconhecimento das complicações, a ausência de verificação dos materiais, a comunicação deficiente entre a equipe, bem como, distrações, condutas de pós-operatório inapropriadas, intervenções e sítios e/ou pacientes incorretos, ocasionam erros que poderiam ser evitados ${ }^{5,6}$.

Diante do contexto exposto, em 2004, com a finalidade de valorizar a segurança do paciente, a OMS desenvolveu a Aliança Mundial para a Segurança do Paciente. Lançado em 2008, o segundo Desafio Global, denominado de Cirurgias Seguras Salvam Vidas, desenvolveu a Lista de Verificação de Segurança Cirúrgica. Essa lista, titulada checklist de cirurgia segura, foi elaborada com base nos dez objetivos indispensáveis para um procedimento seguro. Os propósitos são aperfeiçoar a segurança nas intervenções cirúrgicas e reduzir riscos, eventos adversos, complicações e mortes evitáveis durante o procedimento e no período pós-operatório ${ }^{3}$.

A implementação desse padrão mundial de segurança cirúrgica caracteriza-se como acessível, pois é concreto, claro, de baixo custo, com duração de apenas 3 minutos, não acarreta danos ao paciente e há viabilidade de ser adaptado de acordo com os distintos cenários cirúrgicos ${ }^{1,3}$. Um único profissional, nomeado coordenador, é responsável pelo checklist, porém é imprescindível a cooperação da equipe cirúrgica e do paciente ${ }^{3}$. É recomendado que o instrumento seja coordenado por qualquer profissional que conheça o procedimento, mas que o enfermeiro oriente a execução ${ }^{3}$.

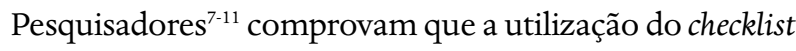
promove progresso na comunicação entre as equipes e diminuição dos erros, complicações e mortes em virtude de procedimentos cirúrgicos, sendo favorável para o paciente, para a equipe e para a unidade de saúde. Uma pesquisa internaciona ${ }^{10}{ }^{10}$ realizada em oito hospitais de oito países com diferentes contextos econômicos teve como resultado queda de $36 \%$ das complicações e de $47 \%$ dos óbitos após a introdução do instrumento, além de ter ocorrido aumento do uso de antibióticos de 56 para $83 \%$, o que resultou em redução das infecções de sítio cirúrgico (ISC).

Em recente estudo ${ }^{11}$ desenvolvido em um hospital geral de grande porte no Rio Grande do Sul, os autores atestaram redução nas taxas de ISC, de 4,2 para 1,1\%, e diminuição de risco identificado de $75 \%$, após uso do checklist. Em outro trabalho ${ }^{9}$, elaborado com 147 enfermeiros de todas as regiões do Brasil, $76,9 \%$ dos profissionais declararam que executam o instrumento na instituição e $83,2 \%$ constataram progresso na assistência. Os que não aplicam o instrumento afirmaram que quando estivessem atuando gostariam que fosse empregado. Mudança na comunicação interprofissional foi relatada por $78,8 \%$ dos enfermeiros. 
Sendo assim, pergunta-se: Qual é a adesão ao checklist de cirurgia segura em procedimentos realizados em crianças e adolescentes até 17 anos, decorridos dez anos do desenvolvimento da Lista de Verificação?

\section{OBJETIVO}

Analisar a adesão ao preenchimento do checklist de cirurgia segura em procedimentos realizados em crianças e adolescentes de até 17 anos, bem como os fatores que influenciam a sua utilização.

\section{MÉTODO}

Trata-se de um estudo analítico, transversal, sobre a adesão do preenchimento do checklist de cirurgia segura pelos profissionais do Centro Cirúrgico (CC) de um hospital público, de pequena e média complexidade, no interior paulista.

O hospital dispõe de quatro salas cirúrgicas, que atendem às especialidades de cirurgia geral, cirurgia pediátrica (cir ped.), cirurgia vascular, urologia (urol.), ginecologia, oftalmologia (oftalmo), otorrinolaringologia (otorrino), ortopedia (ortop.), mastologia, dermatologia e cirurgia plástica. Realizam-se, em média, 200 procedimentos anestésico-cirúrgicos por mês.

Como critério de inclusão dos documentos, neste estudo foram considerados os checklists relativos às cirurgias realizadas em pacientes com idade até 17 anos, 11 meses e 29 dias no CC, no período de janeiro a dezembro de 2017. Os dados das cirurgias foram verificados entre abril e junho de 2018, nos prontuários eletrônicos, por meio de um instrumento desenvolvido pelas pesquisadoras especialmente para o presente estudo.

As variáveis dependentes, ou indicadores da adesão ao checklist, consistem em: existência de checklist no prontuário (sim ou não); preenchimento do checklist (completo, parcial ou não preenchido); preenchimento de cada momento: antes da indução anestésica (momento I - itens 1 ao 7), antes da incisão cirúrgica (momento II — itens 8 ao 14) e antes da saída da sala de operação (momento III - itens 15 ao 19); preenchimento de cada um dos itens; e percentual de itens preenchidos.

O momento I (identificação ou sign in) corresponde ao período anterior à indução anestésica:

1. identificação de dados e consentimento do paciente;

2. sítio cirúrgico demarcado;

3. verificação de segurança anestésica;

4. oxímetro de pulso;
5. alergias;
6. via aérea difícil;
7. risco de perda sanguínea.

Já o momento II (confirmação ou timeout) se refere ao período após a indução anestésica e antes da incisão cirúrgica:

8. apresentação dos membros da equipe;

9. confirmação de dados do paciente pela equipe;

10. duração prevista;

11. revisão do anestesiologista;

12. revisão da equipe de enfermagem;

13. profilaxia antimicrobiana;

14. exames de imagens disponíveis.

Por sua vez, o momento III (registro ou sing out) equivale ao período durante ou imediatamente após o fechamento da ferida e antes de o paciente sair da sala de operação:

15. confirmação do registro e contagem de instrumentos;

16. contagem de compressas;

17. identificação de amostras;

18. problemas com equipamentos;

19. revisão das preocupações para a recuperação.

Ressalta-se que o checklist está inserido no prontuário eletrônico dos pacientes, e não houve divergência do instrumento proposto pela OMS.

As variáveis independentes avaliadas, potencialmente associadas à adesão, foram: dados de caracterização, com a idade do paciente (anos) e o sexo (feminino ou masculino); dados cirúrgicos, incluindo mês (janeiro a dezembro); turno (manhã ou tarde); especialidade médica (cirurgia geral, cirurgia vascular, cir. Ped., urologia, ginecologia, oftalmo, otorrino, ortop., mastologia, dermatologia e cirurgia plástica); cirurgia finalizada; duração da cirurgia (minutos); tipo de anestesia (geral, regional, sedação e/ ou local) e profissional responsável pelo checklist.

Utilizou-se uma amostragem não probabilística, do tipo intencional, incluindo todas as cirurgias realizadas em crianças e adolescentes no ano de 2017. Os dados foram digitados em planilha no Microsoft Excel e, posteriormente, analisados pelos programas Statistical Package for the Social Sciences (SPSS), versão 15.0, e Rv.2.11.0. Foi realizada estatística descritiva com frequência, média e mediana para caracterizar as cirurgias. A associação entre a adesão ao checklist por especialidade médica foi analisada pelo teste exato de Fisher. Com o objetivo de verificar se a duração da cirurgia interfere no preenchimento do instrumento, usaram-se os boxplots. Consideraram-se significativos todos os efeitos e relações associados a valores de $\mathrm{p}<0,05$. 
Esta pesquisa foi aprovada pelo Comitê de Ética em Pesquisa da instituição proponente, sob número do Parecer 2.499.116 e Registro 81985417.8.0000.5411, atendendo aos preceitos de ética em pesquisa, sendo dispensado o Termo de Consentimento Livre e Esclarecido por tratar-se da utilização de dados secundários retrospectivos.

\section{RESULTADOS}

No período do estudo, identificamos 271 cirurgias, no entanto a ausência do checklist no prontuário representou 2,58\% das cirurgias e o não preenchimento $0,7 \%$. Dessa forma, a amostra do estudo foi composta de 262 prontuários de crianças e adolescentes. De acordo com os dados retratados na Tabela 1, 64,9\% dos pacientes são do sexo masculino, com média de idade de 8 anos. O mês com maior número de procedimentos realizados foi maio (14,9\%), com predomínio no período da manhã $(63,7 \%)$. Nos meses de janeiro, novembro e dezembro houve diminuição significativa das cirurgias no serviço, fato que já estava programado. Nesse período, ocorreram somente procedimentos com anestesia local, e as cirurgias de mais complexidade foram encaminhadas para o hospital terciário.

A anestesia apresentou $n=325$, pois se realizou mais de um tipo de procedimento anestésico em um mesmo paciente, e a mais utilizada foi a anestesia geral $(53,9 \%)$. Houve predomínio da especialidade médica de otorrino (35,5\%), seguida por ortop. $(24,0 \%)$ e cir. ped. (16,4\%). Foram realizados 354 procedimentos, em razão de um mesmo paciente ter sido submetido a diferentes intervenções no mesmo ato cirúrgico. Os principais procedimentos executados foram adenoidectomia e amigdalectomia (24,3\%), seguidos por postectomia (8,5\%), com média de 45 minutos de cirurgia.

Em 99,6\% dos formulários avaliados, o profissional responsável foi o técnico de enfermagem e em $0,4 \%$ o enfermeiro. Do preenchimento da Lista de Verificação, em 34 casos (12,9\%), observou-se que os itens estavam preenchidos completamente; em $228(86,4 \%)$ foram preenchidos parcialmente; e dois $(0,7 \%)$ não foram preenchidos. Em relação aos momentos do checklist, a avaliação da adesão à lista ocorreu em 262 instrumentos, pois foram excluídos os que não estavam preenchidos. Verificou-se que o momento I teve $90,4 \%$ de preenchimento completo, o momento II $92,5 \%$ e o momento III $17,1 \%$.

Considerando os 19 itens do checklist, o que apresentou menor adesão no preenchimento foi "Revisão das preocupações para a recuperação”, do momento III, com 77,1\% de inadequação, seguido por "Revisão da equipe de enfermagem", do momento
Tabela 1. Distribuição das cirurgias segundo sexo, idade dos pacientes, mês, turno, anestesia, especialidade, procedimento e duração.

\begin{tabular}{|c|c|c|}
\hline Variáveis & Número & $\begin{array}{c}\text { Percentagem } \\
(\%)\end{array}$ \\
\hline \multicolumn{3}{|l|}{ Sexo } \\
\hline Feminino & 92 & 35,1 \\
\hline Masculino & 170 & 64,9 \\
\hline Idade (anos)* & $8 ; 7(1-17)$ & \\
\hline \multicolumn{3}{|l|}{ Mês } \\
\hline Janeiro & 0 & 0 \\
\hline Fevereiro & 15 & 5,7 \\
\hline Março & 37 & 14,1 \\
\hline Abril & 33 & 12,6 \\
\hline Maio & 39 & 14,9 \\
\hline Junho & 26 & 9,9 \\
\hline Julho & 23 & 8,8 \\
\hline Agosto & 29 & 11,0 \\
\hline Setembro & 23 & 8,8 \\
\hline Outubro & 27 & 10,3 \\
\hline Novembro & 08 & 3,1 \\
\hline Dezembro & 02 & 0,8 \\
\hline \multicolumn{3}{|l|}{ Turno } \\
\hline Manhã & 167 & 63,7 \\
\hline Tarde & 95 & 36,3 \\
\hline \multicolumn{3}{|l|}{ Tipo de anestesia $(n=325)$} \\
\hline Geral & 175 & 53,9 \\
\hline Sedação & 82 & 25,2 \\
\hline Regional & 50 & 15,4 \\
\hline Local & 15 & 4,6 \\
\hline Não informado & 03 & 0,9 \\
\hline \multicolumn{3}{|l|}{ Especialidade cirúrgica } \\
\hline Otorrinolaringologia & 93 & 35,5 \\
\hline Ortopedia/traumatologia & 63 & 24,1 \\
\hline Cirurgia pediátrica & 43 & 16,4 \\
\hline Oftalmologia & 31 & 11,8 \\
\hline Urologia & 23 & 8,8 \\
\hline Outros & 09 & 3,4 \\
\hline \multicolumn{3}{|l|}{ Procedimento cirúrgico $(n=354)$} \\
\hline Adenoidectomia & 86 & 24,3 \\
\hline Amigdalectomia & 86 & 24,3 \\
\hline Postectomia & 30 & 8,5 \\
\hline Hernioplastia umbilical & 18 & 5,1 \\
\hline Outros & 134 & 37,8 \\
\hline Tempo da cirurgia (minutos)* & $45 ; 49(5-170)$ & \\
\hline
\end{tabular}


II, e por "Identificação de dados e consentimento do paciente", do momento I, com $2,8 \%$ das inadequações (Tabela 2).

Não houve associação entre a adesão ao checklist e as principais especialidades cirúrgicas (otorrino, ortop., cir. ped., oftalmo., urologia e outras) nos momentos I ( $\mathrm{p}=0,248)$, II ( $\mathrm{p}=0,895)$ e III $(\mathrm{p}=0,627)$ da Lista de Verificação (Tabela 3$)$.

Verificou-se que, independentemente do momento do checklist ou considerando o processo de preenchimento como um todo, a duração da cirurgia não interferiu para que ocorresse o preenchimento completo, parcial ou o não preenchimento da lista (Figura 1).

Tabela 2. Inadequação do preenchimento dos itens do checklist, de acordo com cada momento da Lista de Verificação.

\begin{tabular}{|c|c|c|}
\hline Item & Número & $\begin{array}{c}\text { Percentagem } \\
(\%)\end{array}$ \\
\hline Momento I & 27 & 9,6 \\
\hline $\begin{array}{l}\text { Identificação de dados e } \\
\text { consentimento do paciente }\end{array}$ & 8 & 2,8 \\
\hline Sítio cirúrgico demarcado & 1 & 0,3 \\
\hline $\begin{array}{l}\text { Verificação de } \\
\text { segurança anestésica }\end{array}$ & 3 & 1,0 \\
\hline Oxímetro de pulso & 2 & 0,8 \\
\hline Alergias & 6 & 2,1 \\
\hline Via aérea difícil & 3 & 1,0 \\
\hline Risco de perda sanguínea & 4 & 1,4 \\
\hline Momento II & 21 & 7,5 \\
\hline $\begin{array}{l}\text { Apresentação dos } \\
\text { membros da equipe }\end{array}$ & 2 & 0,8 \\
\hline $\begin{array}{l}\text { Confirmação de dados } \\
\text { do paciente pela equipe }\end{array}$ & - & - \\
\hline Duração prevista & 2 & 0,8 \\
\hline $\begin{array}{l}\text { Revisão do } \\
\text { anestesiologista }\end{array}$ & 2 & 0,8 \\
\hline $\begin{array}{l}\text { Revisão da equipe } \\
\text { de enfermagem }\end{array}$ & 8 & 2,8 \\
\hline Profilaxia antimicrobiana & 4 & 1,4 \\
\hline $\begin{array}{l}\text { Exames de imagens } \\
\text { disponíveis }\end{array}$ & 3 & 1,0 \\
\hline Momento III & 232 & 82,9 \\
\hline $\begin{array}{l}\text { Confirmação do registro e } \\
\text { contagem de instrumentos }\end{array}$ & 7 & 2,5 \\
\hline Contagem de compressas & 2 & 0,8 \\
\hline Identificação de amostras & 2 & 0,8 \\
\hline $\begin{array}{l}\text { Problemas com } \\
\text { equipamentos }\end{array}$ & 5 & 1,8 \\
\hline $\begin{array}{l}\text { Revisão das preocupações } \\
\text { para a recuperação }\end{array}$ & 216 & 77,1 \\
\hline
\end{tabular}

\section{DISCUSSÃO}

Com a amostra, composta de 262 prontuários analisados, o perfil cirúrgico apresentou prevalência de pacientes do sexo masculino (64,9\%), dado similar aos dos demais estudos ${ }^{5,12}$, que apontaram 70 e $55 \%$ das cirurgias executadas em homens. Em relação à idade prevalente, não foram encontradas pesquisas que abordassem o uso do checklist apenas em procedimentos realizados em crianças e adolescentes, porém uma análise ${ }^{12}$ desenvolvida no Hospital Universitário de Londrina (PR) teve como resultado $20 \%$ dos pacientes com idade menor ou igual a 20 anos.

O propósito da Lista de Verificação de cirurgia segura baseia-se em melhorar a qualidade da assistência cirúrgica e certificar a segurança nos procedimentos anestésico-cirúrgicos, por meio de um padrão que possa ser aplicado mundialmente $^{3}$. O checklist favorece ordenação e padronização dos procedimentos, compartilhamento de informações entre a equipe, com troca de conhecimentos e ansiedades, preparo para possíveis eventos indesejáveis e diminuição do desconforto oriundo de situações inesperadas ${ }^{2,8}$. Desse modo, ocorrem práticas eficazes, melhora na qualidade da comunicação e redução dos riscos, de eventos adversos e de complicações das intervenções cirúrgicas, elevando a segurança do paciente ${ }^{1,2,8}$.

O passo inicial para a segurança cirúrgica consiste na implementação do instrumento de verificação para todos os pacientes cirúrgicos. A presença do checklist no prontuário eletrônico representou $97,4 \%$, porcentagem maior do que a relatada em estudo internaciona ${ }^{13}$, com $83,3 \%$, e nacional ${ }^{14}$, que apontou apenas $60,5 \%$. Inserir o instrumento nas instituições foi considerado de baixo custo econômico, por causa da propagação dele nos prontuários e da sua execução por um profissional, contudo ainda há uma lacuna na instalação do checklist ${ }^{3,9}$. Neste estudo, em sete casos não foi encontrado o instrumento no prontuário, o que reflete uma pequena porcentagem, mas é preciso que a implantação atinja todos os pacientes para, assim, pôr em prática a Lista de Verificação.

O principal profissional responsável pelo checklist foi o técnico de enfermagem, como retratado em pesquisa ${ }^{15}$, atribuindo grande responsabilidade a esses profissionais. Sendo assim, é de suma importância que o enfermeiro participe constantemente de atividades de educação em saúde, a fim de ensinar e reforçar o modo apropriado de realizar a checagem, e encoraje a equipe para pausar o procedimento quando os momentos não estiverem em conformidade. Pesquisadores enfatizam a relevância da comunicação entre os profissionais e destacam que interromper a cirurgia em uma das etapas, dependendo do profissional que faça a interrupção, pode gerar conflitos entre a equipe $e^{3,6,9}$. 
O preenchimento completo do instrumento ocorreu em $12,9 \%$ das cirurgias avaliadas, dificuldade também evidenciada em estudos desenvolvidos em dois hospitais de ensino de Natal $(\mathrm{RN})^{14}$, que demonstraram adesão de apenas 3,5\% das 375 cirurgias ginecológicas e urológicas, e na Espanha ${ }^{13}$, com $27,8 \%$ de adesão no que tange aos 90 procedimentos avaliados em nove hospitais públicos. Já em uma pesquisa ${ }^{5}$ feita em um hospital público de São Paulo analisando 30 procedimentos cardiológicos, $43 \%$ dos instrumentos estavam em conformidade.
Esse déficit na adesão ao checklist, com apenas 12,9\% dos instrumentos com preenchimento completo e $86,4 \%$ com preenchimento parcial, sinaliza a necessidade de ações educativas nas unidades cirúrgicas com toda a equipe, a fim de esclarecer o real entendimento da aplicação do instrumento, que pode favorecer a segurança cirúrgica e a qualidade da assistência prestada, além de incentivar os profissionais sobre sua importância no processo e exibir os resultados positivos após a introdução do protocolo.

Tabela 3. Distribuição das cirurgias segundo as principais especialidades em relação à inadequação dos momentos do checklist.

\begin{tabular}{|c|c|c|c|c|c|c|c|}
\hline & \multicolumn{6}{|c|}{ Especialidades cirúrgicas } & \multirow{2}{*}{$P^{*}$} \\
\hline & Otorrino & Ortop. & Cir. Ped. & Oftalmo. & Urologia & Outras & \\
\hline $\begin{array}{l}\text { Inadequado } \\
\text { Momento I }\end{array}$ & $\begin{array}{c}9 \\
(9,7 \%)\end{array}$ & $\begin{array}{c}7 \\
(11,1 \%)\end{array}$ & $\begin{array}{c}5 \\
(7,0 \%)\end{array}$ & $\begin{array}{c}7 \\
(23,0 \%)\end{array}$ & $\begin{array}{c}1 \\
(4,0 \%)\end{array}$ & $\begin{array}{c}2 \\
(22,2 \%)\end{array}$ & 0,248 \\
\hline $\begin{array}{l}\text { Inadequado } \\
\text { Momento II }\end{array}$ & $\begin{array}{c}4 \\
(4,3 \%)\end{array}$ & $\begin{array}{c}6 \\
(6,3 \%)\end{array}$ & $\begin{array}{c}2 \\
(4,7 \%)\end{array}$ & $\begin{array}{c}1 \\
(3,2 \%)\end{array}$ & $\begin{array}{c}0 \\
(0,0 \%)\end{array}$ & $\begin{array}{c}1 \\
(11,1 \%)\end{array}$ & 0,895 \\
\hline $\begin{array}{l}\text { Inadequado } \\
\text { Momento III }\end{array}$ & $\begin{array}{c}78 \\
(83,9 \%)\end{array}$ & $\begin{array}{c}53 \\
(84,1 \%)\end{array}$ & $\begin{array}{c}37 \\
(86,0 \%)\end{array}$ & $\begin{array}{c}24 \\
(77,4 \%)\end{array}$ & $\begin{array}{c}21 \\
(91,3 \%)\end{array}$ & $\begin{array}{c}07 \\
(77,8 \%)\end{array}$ & 0,627 \\
\hline
\end{tabular}

*Teste exato de Fisher.

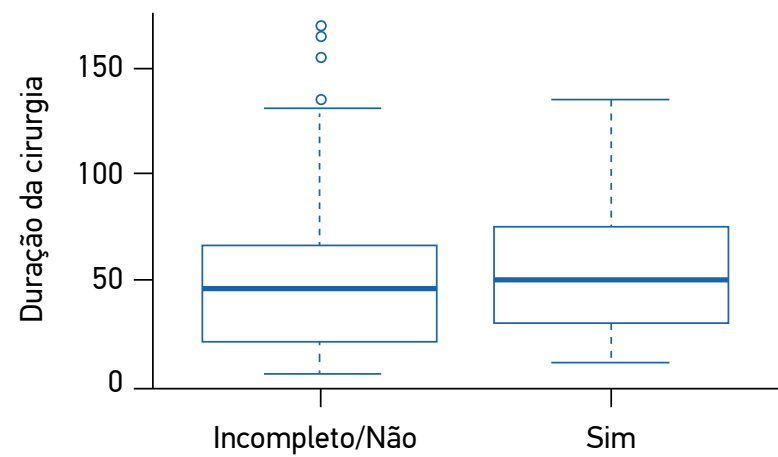

Preenchimento do checklist

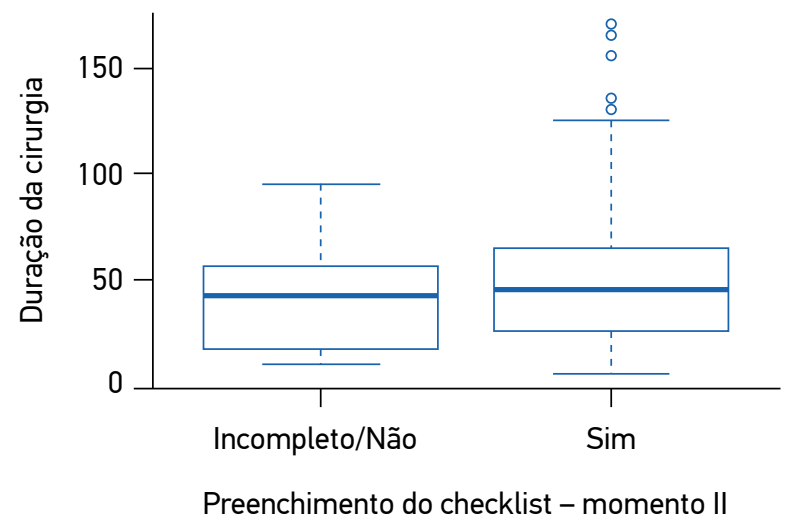

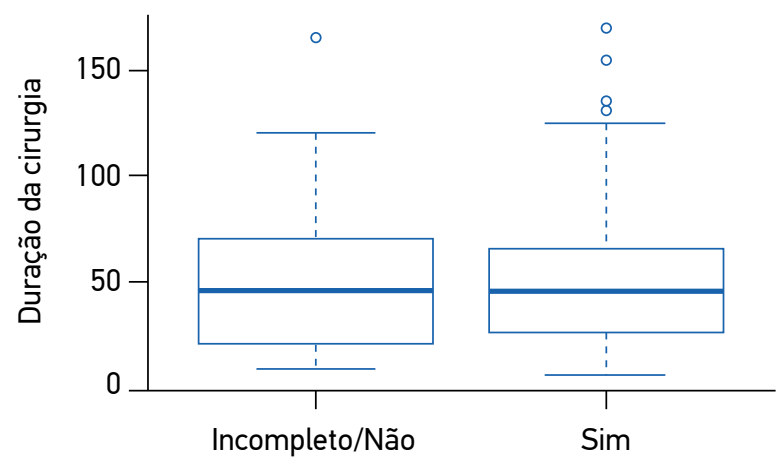

Preenchimento do checklist - momento I

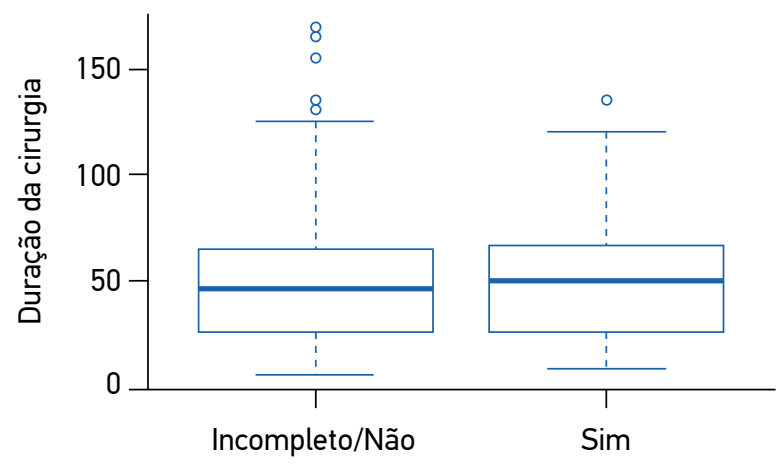

Preenchimento do checklist - momento III

Figura 1. Boxplot da duração da cirurgia (em minutos) segundo o preenchimento do checklist. Botucatu, Brasil, 2018. 
Considerando os três momentos, o que teve maior adesão neste estudo foi o momento II, referente à confirmação, com $92,5 \%$ do preenchimento completo, corroborando com pesquisa espanhola ${ }^{13}$, que apresentou $51,8 \%$. Em seguida, a identificação, relacionada ao primeiro momento, teve como resultado 90,4\%, e na Espanha ${ }^{13}$, 49,3\%. O momento com menor adesão foi o terceiro, denominado de Registro, com apenas $17,1 \%$, contrastando com $43,1 \%$ do estudo espanhol ${ }^{13}$ e com pesquisa ${ }^{15}$ realizada em Belo Horizonte (MG), que mostrou adesão inferior a $50 \% \mathrm{em}$ todos os itens desse momento. Houve divergência com estudo nacional ${ }^{14}$, que evidenciou o momento I com menor preenchimento. A análise dos momentos com outras pesquisas ${ }^{5,16,17}$ foi dificultada por conta da modificação que algumas instituições efetuaram na Lista de Verificação. Desse modo, havia quatro etapas divergindo do nosso instrumento.

Estudo ${ }^{13}$ aponta como causas da menor adesão ao momento III a exaustão dos profissionais e a ausência do cirurgião responsável. Esse momento não deixa de ser menos importante do que os outros; pelo contrário, aborda questões relevantes, como a contagem de compressas e instrumentais, pois a retenção desses objetos ainda ocorre em grande proporção ${ }^{3,18}$. Em pesquisa ${ }^{18}$ realizada com 2.872 médicos, foram relatados 4.547 casos de retenção inadvertida de corpos estranhos em procedimento cirúrgicos, sendo $68 \%$ compressas grandes e pequenas, $22 \%$ gazes e $5 \%$ instrumentais cirúrgicos.

Dos 19 itens avaliados, o nomeado "Revisão das preocupações para a recuperação" correspondeu a 77,1\% não preenchido, com menor adesão ao momento III. Esse dado demonstra a atenção focada apenas no momento do procedimento, gerando déficit no seguimento do cuidado na recuperação anestésica e no pós-operatório, que pode influenciar na integralidade do cuidado, fragmentando a assistência ao paciente cirúrgico. Em contrapartida, em outros estudos, os itens que tiveram menor adesão foram "Sítio cirúrgico demarcado"14 e "Contagem de gazes"17.

O tempo cirúrgico e as especialidades não influenciaram na adesão ao checklist. Esses dados indicam que não existe uma única causa para a inadequação do instrumento, sendo essencial a cooperação de todos para a execução adequada do instrumento, e as atividades de educação em saúde devem ser realizadas no serviço e envolver todos os profissionais. A literatura destaca que a ausência de apoio das chefias, o desconhecimento do protocolo de segurança cirúrgica, a ausência de treinamento para a equipe, a comunicação deficiente e a rejeição por parte de alguns profissionais em responder verbalmente aos itens a serem checados foram os principais problemas elencados para a não adesão ${ }^{6-9,19}$. Também foi encontrado um estudo ${ }^{8}$ em que os profissionais relataram o tempo como empecilho para execução do instrumento, por conta da sobrecarga do trabalho.

Pesquisadores consideram que a falta de participação da equipe no ato do checklist reflete a cultura do trabalho individual, que, infelizmente, ainda está presente nos dias de hoje, assim como a banalização do instrumento, questões que se tornam limitações para adesão ao checklist ${ }^{6-7,19}$. Estudo $^{15}$ avaliando 30 procedimentos destacou que em nenhum dos checklists houve atuação da equipe multiprofissional, e outro trabalho ${ }^{16}$ identificou que grande parte das verificações ocorreu de maneira individual e não verbal. O trabalho independente prejudica a comunicação entre a equipe, podendo ocorrer conflitos e desgastes, e, dessa maneira, interfere no ambiente de trabalho e no resultado da assistência prestada ${ }^{6,19}$.

Considera-se um fator importante para adesão ao checklist a cultura de segurança do paciente inserida na equipe multiprofissional, com início desde a formação dos profissionais, com ações de educação continuada e permanente para toda a equipe. Estudo ${ }^{20}$ mostrou aumento de 7,9 para $96,9 \%$ na verificação correta do checklist após treinamentos envolvendo a equipe cirúrgica. Sendo assim, a capacitação dos profissionais teve resultados positivos, com impacto na assistência prestada. Em contrapartida, posteriormente a uma capacitação realizada em um hospital público de ensino, houve redução dos protocolos em branco, contudo ampliou-se a quantidade de instrumentos incompletos ${ }^{12}$.

Desse modo, as ações educativas podem ser favoráveis ou desfavoráveis à adesão ao checklist, dependendo do método, da duração e dos temas estipulados, assim como dos profissionais participantes ${ }^{7}$. A metodologia de problematização abordada em pesquisa ${ }^{19}$ reproduz uma estratégia interessante para o treinamento na unidade cirúrgica, pois estimula a participação efetiva de todos os membros da equipe. Primeiramente, estes observam a realidade e reconhecem as dificuldades enfrentadas no dia a dia. Em seguida, as intervenções são elaboradas, por meio de uma análise em conjunto, com base nas origens dos problemas e no porquê de eles ocorrerem. Por fim, pratica-se o proposto, buscando mudanças na realidade de trabalho da equipe multiprofissional.

O presente estudo apresenta como limitação a utilização de dados secundários, no entanto avaliar a adesão ao checklist é o primeiro passo para, posteriormente, analisar o impacto na qualidade da assistência prestada. Nesse sentido, novos estudos devem ser realizados. 


\section{CONCLUSÃO}

A Lista de Verificação de cirurgia segura representa um padrão mundial de segurança cirúrgica, prevenindo erros, eventos adversos, complicações e até mortes na assistência operatória, no entanto são necessárias conscientização e participação das equipes cirúrgicas para aplicar o instrumento adequadamente e, assim, garantir segurança ao paciente.

A adesão completa ao checklist foi de $12,9 \%$ das cirurgias avaliadas. O preenchimento diferiu entre as etapas, com menor adesão ao momento III. O preenchimento parcial, na maioria dos casos, sinaliza a necessidade de se desconstruir as barreiras para essa adesão mediante o empoderamento da equipe de enfermagem para conduzir o checklist, ações educativas com as equipes e o real entendimento da aplicação do instrumento, que pode favorecer a segurança cirúrgica e elevar a qualidade da assistência prestada. Tempo cirúrgico e especialidade não influenciaram na adesão à aplicação da Lista de Verificação, o que indica que não existe uma única causa que explique a inadequação do preenchimento do instrumento.

\section{REFERÊNCIAS}

1. Santos JS, Souza DO, Morais AC, Santana CLM, Rodrigues US, Rodrigues EP. Teste piloto de checklist de cirurgia segura: relato de experiência. Rev Enferm UFPI. 2017;6(1):76-9. https://doi.org/10.26694/reufpi.v6i1.5648

2. MafraCR, Rodrigues MCS. Lista de verificação de segurança cirúrgica: uma revisão integrativa sobre benefícios e sua importância. 2018;10(1):26875. https://doi.org/10.9789/2175-5361.2018.v10i1.268-275

3. Brasil. Ministério da Saúde(MS). Agência Nacional de Vigilância Sanitária (ANVISA). Organização Mundial da Saúde (OMS). Segundo desafio global para a segurança do paciente. Cirurgias seguras salvam vidas. Rio de Janeiro: Organização Pan-Americana da Saúde (OPAS); 2009.

4. Tostes MFP, Haracemiw A, Mai LD. Lista de verificação de segurança cirúrgica: considerações a partir da micropolítica institucional. Esc Anna Nery. 2016;20(1):203-9. http://dx.doi.org/10.5935/1414-8145.20160027

5. Giannattasio MB, Taniguchi FP. Avaliação da segurança do paciente em cirurgia cardíaca de um hospital público. Rev SOBECC. 2016;21(3):12531. https://doi.org/10.5327/Z1414-4425201600030002

6. Gomes CDPP, Santos AA, Machado ME, Treviso P. Percepção de uma equipe de enfermagem sobre a utilização do checklist cirúrgico. Rev SOBECC. 2016;21(3):140-5. https://doi.org/10.5327/ Z1414-4425201600030004

7. Tostes MFP, Galvão CM. Lista de verificação de segurança cirúrgica: benefícios, facilitadores e barreiras na perspectiva da enfermagem. Rev Gaúcha Enferm. 2019;40(núm. esp.):e20180180. https://doi. org/10.1590/1983-1447.2019.20180180

8. Silva FAA, Silva AGN. Equipe de enfermagem em cirurgia segura: desafios para adesão ao protocolo. Rev Enferm UFPI. 2017;6(2):239. https://doi.org/10.26694/reufpi.v6i2.5844

9. Souza RM, Araújo MGS, Veríssimo RCSS, Comassetto I, Ferreira FAS, Bernardo THL. Aplicabilidade do checklist de cirurgia segura em centros cirúrgicos hospitalares. Rev SOBECC. 2016;21(4):1927. https://doi.org/10.5327/Z1414-4425201600040003

10. Haynes AB, Weiser TG, Berry WR, Lipsitz SR, Breizat AH, Dellinger $E P$, et al. A surgical safety checklist reduces morbidity and mortality in a global population. N Engl J Med. 2009;360(5):491-9. https://doi. org/10.1056/NEJMsa0810119
11. Prates CG, Stadñik CM, Bagatini A, Caregnato RC, Moura GM. Comparação das taxas de infecção cirúrgica após implantação do checklist de segurança. Acta Paul Enferm. 2018;31(2):116-22. http:// dx.doi.org/10.1590/1982-0194201800018

12. Elias ACGP, Schmidt DRC, Yonekura CSI, Dias AO, Ursi ES, Silva RPJ, et al. Avaliação da adesão ao checklist de cirurgia segura em hospital universitário público. Rev SOBECC. 2015;20(3):128-33. https://doi. org/10.5327/Z1414-4425201500030002

13. Soria-Aledo V, Silva ZA, Saturno PJ, Grau-Polan M, Carrilo-Alcaraz A. Dificultades en la implantación del checklist en los quirófanos de cirugía. Cir Esp. 2012;90(3):180-5. https://doi.org/10.1016/j.ciresp.2011.09.007

14. Freitas MR, Antunes AG, Lopes BNA, Fernandes FC, Monte LC, Gama ZAS. Avaliação da adesão ao checklist de cirurgia segura da OMS em cirurgias urológicas e ginecológicas, em dois hospitais de ensino de Natal, Rio Grande do Norte, Brasil. Cad Saúde Pública. 2014;30(1):137-48. http://dx.doi.org/10.1590/0102-311X00184612

15. Oliveira AC, Abreu AR, Almeida SS. Implementação do checklist de cirurgia segura em um hospital universitário. Enferm Foco. 2017;8(4):14-8. https://doi.org/10.21675/2357-707X.2017.v8.n4.972

16. Maziero ECS, Silva AEBC, Mantovani MF, Cruz EDA. Adesão ao uso de um checklist cirúrgico para segurança do paciente. Rev Gaúcha Enferm. 2015;36(4):14-20. http://dx.doi.org/10.1590/1983-1447.2015.04.53716

17. Amaya MR, Maziero ECS, Grittem L, Cruz EDA. Análise do registro e conteúdo de checklists para cirurgia segura. Esc Anna Nery. 2015;19(2):246-51. http://dx.doi.org/10.5935/1414-8145.20150032

18. Birolini DV, Rasslan S, Utiyama EM. Retenção inadvertida de corpos estranhos após intervenções cirúrgicas. Análise de 4547 casos. Rev Col Bras Cir. 2016;43(1):2-17. http://dx. doi. org/10.1590/0100-69912016001004

19. Santos KCB, Feitosa AHC, Ribeiro GSC, Cavalcante TB. Metodologia da problematização com Arco de Maguerez no centro cirúrgico oftalmológico de um hospital universitário. Reon Facema. 2018;4(1):884-8.

20. Sewell M, Adebibe M, Jayakumar P, Jowett C, Kong K, Vemulapalli K, et al. Use of WHO surgical safety checklist in trauma and orthopedic patients. Int Orthop. 2011;35(6):897-901. https://doi.org/10.1007/s00264-010-1112-7 